\title{
Desenvolvimento e aplicação de um novo índice para avaliação do pré-natal
}

\author{
Esther Pereira da Silva, ${ }^{1}$ Roberto Teixeira Lima, ${ }^{1}$ \\ Maria José de Carvalho Costa ${ }^{1}$ e Malaquias Batista Filho ${ }^{2}$
}

Como citar

Silva EP, Lima RT, Costa MJC, Batista Filho M. Desenvolvimento e aplicação de um novo índice para avaliação do pré-natal. Rev Panam Salud Publica. 2013;33(5):356-62.

RESUMO Objetivo. Desenvolver e aplicar um novo instrumento para avaliar o pré-natal com base nas diretrizes do Programa de Humanização do Pré-Natal e do Nascimento do Ministério da Saúde brasileiro, inserindo elementos quanti-qualitativos distribuídos segundo a tríade avaliativa de infra-estrutura, processo de trabalho e resultados.

Métodos. Este estudo transversal, de natureza analítico-descritiva e abordagem quantitativa, foi desenvolvido com 238 usuárias em 44 serviços da atenção primária à saúde do Município de João Pessoa, Nordeste do Brasil, de novembro de 2010 a dezembro de 2011. Também foram entrevistados médicos e enfermeiros envolvidos no cuidado pré-natal. O instrumento elaborado pelos pesquisadores continha 23 questões relativas a infra-estrutura, processo de trabalho e resultados da assistência pré-natal. A partir das informações coletadas, o pré-natal foi classificado pelo que denominamos de Índice IPR/Pré-Natal. Para cada questão atribui-se o valor 1 para adequado, quando em conformidade com os critérios estabelecidos, e 2 para inadequado. Com base na porcentagem representada pela soma do número de questões adequadas dos três componentes em relação ao total de 23 questões, o cuidado pré-natal foi classificado em: adequado superior (100\% de questões adequadas); adequado ( $\geq 75 \%$ ); intermediário (51 a $74 \%)$; e inadequado ( $\leq 50 \%$ ). As categorias de classificação do IPR/Pré-Natal foram comparadas com as dos indices de Kessner e Adequacy of Prenatal Care Utilization (APNCU).

Resultados. As questões referentes ao processo de trabalho foram as que contribuíram com significância estatística para que o pré-natal fosse classificado em maior proporção na categoria intermediário pelo Índice IPR/Pré-Natal. As categorias de classificação do Índice IPR/ Pré-Natal mostraram-se consistentes para detectar as variáveis prematuridade, peso insuficiente ao nascer e não realização do aleitamento materno exclusivo.

Conclusões. O Índice IPR/Pré-Natal foi eficaz na incorporação de elementos quanti-qualitativos para classificação do cuidado pré-natal.

Palavras-chave Cuidado pré-natal; atenção primária à saúde; peso ao nascer; nascimento prematuro; aleitamento materno; Brasil.

No período pré-natal, o início precoce do acompanhamento à gestante está relacionado a benefícios tanto para a mãe

Universidade Federal da Paraíba, Centro de Ciências da Saúde, Pós-Graduação em Ciências da Nutrição, João Pessoa (PB), Brasil. Correspondência: Esther Pereira da Silva, estherp.silva@yahoo.com.br

2 Instituto Materno-Infantil Professor Fernando Figueira, Diretoria de Pesquisa, Recife (PE), Brasil. quanto para o bebê, diminuindo a incidência de baixo peso, peso insuficiente ao nascer e prematuridade e promovendo a boa prática de aleitamento materno. A literatura mostra uma associação entre a adequação do cuidado pré-natal e os resultados do parto. Para isso, normalmente, são utilizados índices que verificam a qualidade do cuidado pré-natal
$(1,2)$, como por exemplo os índices de Kessner e de Adequação da Utilização do Cuidado Pré-Natal (Adequacy of Prenatal Care Utilization, APNCU). Além de classificar o cuidado no período pré-natal, esses índices também avaliam a associação entre atenção ao pré-natal e resultados adversos do nascimento, como prematuridade e baixo peso ao nascer (3-5). 
O índice de Kessner categoriza o prénatal como adequado, intermediário e inadequado com base no trimestre de início do acompanhamento e no número de consultas ajustado para idade gestacional. O APNCU acrescenta a categoria adequado superior/intensivo, além de analisar a relação entre o número real e o número esperado de consultas. Quanto ao número de consultas, ambos os índices seguem as recomendações do Colégio Americano de Obstetrícia e Ginecologia (ACOG), recomendando nove e 13 consultas, respectivamente (5-7).

Na avaliação da atenção ao pré-natal, o foco em aspectos quantitativos como o número de consultas e o trimestre inicial pode encobrir a visualização de outros aspectos relevantes para os resultados do nascimento, sendo necessária a inserção de novos componentes que mensurem a qualidade de forma integral $(2,8)$. Nessa temática, Donabedian (9) propõe um modelo de avaliação da qualidade da assistência baseado na tríade estrutura-processo-resultado, que permite identificar com mais propriedade fatores que contribuem para inadequação, buscando a melhoria das ações em saúde (10-12). Há países em que esse tipo de avaliação já é uma prática institucionalizada em outros contextos, com resultados que contribuem para a formulação de políticas e práticas de saúde $(8,13,14)$. Uma tal avaliação poderia ser vantajosamente aplicada ao contexto da atenção pré-natal.

Para ampliar a qualidade do cuidado pré-natal, implantou-se no Brasil, no ano 2000, o Programa de Humanização do Pré-Natal e do Nascimento (PHPN) pelo Ministério da Saúde. O PHPN propõe os seguintes critérios: realização de seis consultas de pré-natal para gestações a termo, sendo a primeira no primeiro trimestre; imunização contra o tétano; exames laboratoriais básicos (incluindo HIV e sífilis); procedimentos clínicoobstétricos; e consulta puerperal (42 dias pós-parto) (15). Essas recomendações já foram utilizadas por estudiosos brasileiros para caracterizar e avaliar a atenção ao pré-natal, principalmente com foco em elementos específicos: indicadores de cobertura, recursos materiais, protocolos de tratamento, satisfação das usuárias e resultados da atenção (16-18).

Quanto ao número de consultas, esse aspecto difere consideravelmente em diferentes países. Por exemplo, na Holanda, assim como no Brasil, são seis as consultas de pré-natal, enquanto que na Finlândia são 15. Na Bélgica, são recomendadas 10 consultas para primíparas e sete para multíparas (19-21). Contudo, internacionalmente, há unanimidade entre pesquisadores quanto à importância de não restringir o cuidado prénatal a aspectos quantitativos. Ressaltase ainda a escassez de um parâmetro validado que racionalize questões da tríade estrutura-processo-resultado em um único instrumento para categorizar a qualidade do pré-natal $(2,8)$.

Nesse contexto, o presente estudo teve como objetivo principal o desenvolvimento e a aplicação de um novo instrumento para avaliar o pré-natal de forma mais abrangente, denominado Índice IPR/Pré-Natal. Esse novo índice proposto se baseia nas diretrizes do PHPN, inserindo elementos quanti-qualitativos distribuídos segundo a tríade avaliativa de infra-estrutura, processo de trabalho e resultados. Além disso, a fim de verificar a validade do instrumento, isto é, se sua faixa de adequação do pré-natal reflete significativamente as categorias que se destina a descrever, foram comparadas suas categorias de classificação com as dos índices usualmente utilizados (Kessner e APNCU) quando associados a desfechos do nascimento (baixo peso e peso insuficiente ao nascer, prematuridade e não realização do aleitamento materno exclusivo).

\section{MATERIAIS E MÉTODOS}

Este estudo transversal, de natureza analítico-descritiva e abordagem quantitativa, foi desenvolvido em serviços da atenção primária à saúde do Município de João Pessoa, capital do Estado da Paraíba. João Pessoa situa-se na Região Nordeste do Brasil. O estudo foi realizado de novembro de 2010 a dezembro de 2011.

Os serviços inseridos no estudo são responsáveis por acolher as gestantes durante o pré-natal e, ao detectar quaisquer riscos à mãe ou ao feto, encaminhálas para acompanhamento por serviços de maior complexidade. No ano anterior à pesquisa, foram atendidas, em João Pessoa, 3362 gestantes, assistidas por 180 unidades básicas de saúde (UBS) distribuídas em cinco distritos sanitários, de acordo com a localização geográfica (18).

$\mathrm{O}$ cálculo para determinar a amostra das UBS e o número de usuárias baseouse nas recomendações de Bolfarine et al.
(22) para amostragem por conglomerado de segundo estágio, conforme a equação

$$
n=1 /\left(\mathrm{D} /(\mathrm{p} . \mathrm{q})^{2}+1 / \mathrm{N}\right),
$$

na qual p é a proporção de UBS com atendimento adequado (no caso, admitiuse $50 \%$, ou 0,5$)$, q é igual a $1-$ p, que é a proporção de UBS com atendimento inadequado, $\mathrm{D}$ é igual a $\mathrm{E}^{2} / \mathrm{z}_{\alpha}{ }^{2}$, onde $\mathrm{E}$ é a margem de erro e $z_{\alpha}$ é o escore da distribuição normal padrão para uma significância de 5\% (ou confiança de 95\%); $\mathrm{N}$ indica o universo da população e $n$ é a amostra da população.

A partir desse cálculo, a amostra foi estabelecida em 44 UBS. Para determinar o número da amostra de usuárias, atribuiu-se aos valores de $p$ e $q$ o percentual de gestantes que fizeram ou não fizeram, respectivamente, seis ou mais consultas de pré-natal no ano anterior no Município, conforme dados obtidos no Sistema de Informação da Atenção Básica do Brasil (SIAB) (18). Assim, obteve-se o total de 254 usuárias (todas gestantes que durante o estudo tornaram-se mães). A amostra final resultou em 238 usuárias (perda de 6,30\%), pois 16 apresentaram ausência de informações, sendo o número obtido suficiente para proporcionar análises consistentes. Em cada UBS, por amostra de conveniência, foram entrevistados os profissionais mais envolvidos diretamente no cuidado pré-natal (médicos e/ ou enfermeiros).

Às gestantes, a data provável do parto era solicitada para posterior visita aos serviços e coleta de dados obstétricos (peso ao nascer do neonato, idade gestacional do parto e aleitamento materno). Foram fatores de exclusão: gravidez de alto risco, casos em que o pré-natal é feito por serviços de maior complexidade, e data de parto fora do período da pesquisa. Para a análise do aleitamento materno exclusivo, considerou-se apenas as mães de crianças com no mínimo 6 meses de idade. Para essa variável, portanto, foram analisadas 128 usuárias.

A coleta de dados foi desenvolvida por meio de um instrumento específico elaborado pelos pesquisadores do estudo. Este se apresenta estruturado em três eixos de análise, conforme recomenda Donabedian (9): infra-estrutura, processo de trabalho e resultados da assistência pré-natal. Para os dois primeiros, seis questões foram desenvolvidas. Para o componente resultados, 11 questões foram elaboradas. 
As informações referentes à infraestrutura e ao processo de trabalho foram obtidas a partir de entrevistas aos profissionais durante as visitas. Quanto aos resultados da assistência pré-natal, os dados foram coletados por meio de entrevistas às usuárias e consultas a seus registros no Cartão da Gestante e Caderneta da Criança; esses são documentos oficiais preenchidos pelos profissionais de saúde dos serviços durante as consultas, apresentando dados quanto à assistência ao pré-natal e parto, considerados fontes válidas e seguras de informações para pesquisas científicas (23).

A partir das informações coletadas por esse instrumento, o pré-natal foi classificado pelo que denominamos de Índice IPR/Pré-Natal, com IPR correspondendo a infra-estrutura (I), processo (P) e resultados (R). Para cada questão de cada um dos componentes, atribui-se o valor 1 para adequado, quando em conformidade com os critérios estabelecidos, e 2 para inadequado, conforme descrição abaixo.

$\mathrm{Na}$ infra-estrutura, as seis questões de análise classificadas como adequadas (valor $=1$ ) referiam-se a serviços que apresentavam: instalação em prédio construído para o fim específico (sem adaptação de outras construções), identificação explícita dos dias e horários de pré-natal, apoio para exames laboratoriais (no local ou de referência), equipamentos, recursos materiais (higiênico-sanitários e para gestão da informação) e medicamentos/suplementos importantes para o pré-natal segundo recomendações do PHPN (23).

Para o processo de trabalho, as seis questões de análise obtinham o valor 1 quando os serviços apresentavam-se em conformidade com os seguintes critérios: cobertura da população de gestantes acompanhadas e gestantes que cumpriram as metas propostas pelo PHPN acima de $90 \%$, ter multidisciplinaridade nas consultas (com mais de um profissional de nível superior), realizar os procedimentos clínico-obstétricos da rotina do pré-natal recomendados pelo PHPN, realizar pelo menos uma atividade de educação em saúde de interesse no pré-natal e apresentar no mínimo um indicador que avaliasse internamente a atenção ao pré-natal no serviço.

Nas 11 questões do componente de resultados do cuidado pré-natal, o valor 1 era atribuído quando: a primeira consulta das usuárias acontecia no pri- meiro trimestre, havendo no mínimo seis consultas ao longo do pré-natal, uso de suplementos via oral de micronutrientes essenciais à gestação, consultas multiprofissionais de pré-natal (com a presença de mais de um profissional de nível superior), exames laboratoriais com resultados registrados no cartão da gestante e procedimentos clínico-obstétricos segundo as normas do PHPN (23), ter tido pelos profissionais de saúde orientações quanto ao aleitamento materno durante a gestação, participação em no mínimo uma atividade de educação em saúde extra-consulta pré-natal, ganho de peso gestacional em conformidade com normas internacionais (24), usuária sem intercorrências clínicas ao longo da gestação e consulta puerperal no pós-parto.

As três dimensões avaliadas pelo índice foram ponderadas com igual escore de importância, ao considerar a relação causal existente entre elas, que proporciona a qualidade (9). A classificação do pré-natal por este método foi feita com base na porcentagem representada pela soma do número de questões adequadas dos três componentes avaliados em relação ao total de 23 questões do instrumento. A partir deste cálculo, o cuidado pré-natal foi classificado em quatro categorias: adequado superior, quando $100 \%$ das questões receberam o valor 1 (adequado); adequado, quanto os itens adequados representaram $75 \%$ ou mais das respostas; intermediário, de 51 a $74 \%$ de respostas adequadas; e inadequado, quando $50 \%$ ou menos das respostas eram adequadas.

Foi realizado o procedimento do préteste em serviços não-amostrais, no qual foram identificadas perguntas que exigiam alterações na redação ou no formato, ou questões que acabaram eliminadas na versão final. Após esse procedimento, o instrumento proposto foi considerado finalizado quando atingiu um nível homogêneo nas respostas. $\mathrm{O}$ treinamento dos sujeitos para aplicação do instrumento foi conduzido sob orientação de professores universitários e profissionais de nível superior envolvidos em todas as etapas da construção do questionário, a fim de estabelecer homogeneidade na coleta das informações.

As características da população do estudo, atenção ao pré-natal e desfechos do nascimento foram apresentados por meio de mediana (percentis 25 e 75), média, desvio-padrão e amplitude. $\mathrm{O}$ teste de correlação de Spearman foi realizado para verificar qual dos três componentes do índice proposto foi mais sensível e específico para classificar o pré-natal na categoria de maior inadequação.

Para verificar a consistência de classificação do novo instrumento, foi realizada a análise comparativa interna das categorias de adequação do mesmo com os índices já validados de Kessner e APNCU. Para a análise comparativa, foram utilizados modelos para quatro resultados de nascimento diferentes: baixo peso ao nascer, peso insuficiente ao nascer, prematuridade e não realização do aleitamento materno exclusivo (25). Cada um dos modelos foi ajustado para variáveis de confundimento: características sócio-demográficas das gestantes (idade, raça, escolaridade, estado civil) e dados clínicos (tabagismo, ingesta de álcool, se a mãe teve um parto prematuro anterior e se a mãe já tinha dado à luz um bebê pesando mais de $4000 \mathrm{~g}$ ).

Para cada um dos quatro desfechos de nascimento, três modelos diferentes foram usados, um para cada um dos três índices avaliados. Para todos os casos, a razão de chances (odds ratio, OR) ajustada e o respectivo intervalo de confiança (IC) foram calculados para comparação das categorias de adequação dos índices utilizando a categoria adequado como referência.

A fim de verificar a validade do novo instrumento, examinou-se, para cada desfecho de nascimento e para cada índice de pré-natal, se o padrão de OR ajustado correspondeu ao que seria de esperar se as categorias de adequação dos índices realmente descrevessem o que pretendem, isto é: se a categoria adequada contivesse, efetivamente, o nível de assistência suficiente às necessidades da gravidez.

Para determinadas variáveis, não foi possível o cálculo da OR quando $100 \%$ dos casos encontravam-se em uma única categoria, obtendo no cruzamento das caselas o valor 0 . A significância considerada foi de $5 \%$. Os dados foram gerenciados e analisados pelos programas estatísticos R versão 2.10.1 e EPI-Info versão 6.04 .

A pesquisa seguiu os preceitos éticos referidos na declaração de Helsinki e na Resolução 196/96 do Ministério da Saúde do Brasil. O projeto foi aprovada pelo Comitê de Ética em Pesquisa do Hospital Universitário Lauro Wanderley, da Universidade Federal da Paraíba (protocolo 660/2010). 
TABELA 1. Características da população do estudo, atenção ao pré-natal e resultados do nascimento, João Pessoa (PB), Brasil, 2012

\begin{tabular}{|c|c|c|c|}
\hline Características da população $(n=238)$ & Média \pm DP & Mediana (P25-P75) & Amplitude \\
\hline Idade da mãe (anos completos) & $24,19 \pm 4,19$ & $24(22$ a 26$)$ & 15 (18 a 33) \\
\hline Escolaridade (anos de estudo) & $9,35 \pm 2,11$ & $8(7$ a 10) & 9 (3 a 12) \\
\hline Paridade & $1,79 \pm 0,91$ & $2(1 \mathrm{a} 2)$ & $5(1$ a 6$)$ \\
\hline \multicolumn{4}{|l|}{ Ganho de peso gestacional } \\
\hline Eutrófico & $12,295 \pm 1,45$ & $12(10$ a 14$)$ & $7,6(17,2$ a 9,6$)$ \\
\hline Sobrepeso & $12,612 \pm 1,82$ & $12(10$ a 14$)$ & $8,1(9,1$ a 17,2$)$ \\
\hline Obesidade & $13,170 \pm 3,07$ & $13(10$ a 16$)$ & $7,2(7$ a 17,2$)$ \\
\hline Idade gestacional de início do pré-natal & $11,27 \pm 3,37$ & $11(10$ a 12) & 17 (5 a 22) \\
\hline Total de consultas de pré-natal & $7,64 \pm 1,59$ & $6(5$ a 8$)$ & 9 (4 a 13) \\
\hline \multicolumn{4}{|l|}{ Resultados do nascimento } \\
\hline Idade gestacional do parto & $38,26 \pm 0,98$ & $38(37$ a 39) & $5(36$ a 41) \\
\hline Peso ao nascer $(\mathrm{g})$ & $3204,91 \pm 486,43$ & 3 082,50 (2 980 a 3 421) & 2071 (2 419 a 4 490) \\
\hline Tempo de aleitamento materno exclusivo (dias) ${ }^{a}$ & $58,05 \pm 34,19$ & $60(60$ a 94$)$ & $150(30$ a 180) \\
\hline
\end{tabular}

TABELA 2. Classificação do cuidado pré-natal em 238 mulheres de acordo com três índices de avaliação, João Pessoa (PB), Brasil, 2012

\begin{tabular}{lccc}
\hline \multirow{2}{*}{ Classificação } & \multicolumn{3}{c}{ Indice de avaliação } \\
\cline { 2 - 4 } \multicolumn{1}{c}{ IPR/Pré-Natal (\%) } & Kessner (\%) & APNCU (\%) \\
\hline Adequado superior/intensivo & 0,00 & $-^{\text {a }}$ & 6,72 \\
Adequado & 34,54 & 42,44 & 26,05 \\
Intermediário & 65,46 & 54,62 & 63,87 \\
Inadequado & 0,00 & 2,94 & 3,36 \\
Sem assistência/ desconhecido & 0,00 & 0,00 & 0,00 \\
\hline
\end{tabular}

IPR: infra-estrutura, processo e resultados. APNCU: Adequação da Utilização do Cuidado Pré-Natal.

${ }^{a}$ Categoria inexistente no índice.

\section{RESULTADOS}

A tabela 1 apresenta as características da população. O ganho de peso gestacional foi maior nas mulheres com índice de massa corporal (IMC) prégestacional maior. A maior parte das gestantes iniciou o pré-natal no primeiro trimestre, com mediana de seis consultas realizadas. Além disso, a maioria das gestantes apresentou gestação a termo, com peso adequado na maior parte dos bebês (média de $3204,91 \pm$ $486,43 \mathrm{~kg}$ ). O tempo de aleitamento materno exclusivo foi baixo.

A tabela 2 descreve o percentual de adequação do pré-natal da população de acordo com cada índice. $\mathrm{O}$ índice de Kessner resultou na maior proporção de casos na categoria adequado. O Índice IPR/Pré-Natal foi o procedimento que classificou o maior número de casos na categoria intermediário. A classificação do cuidado pré-natal como adequado superior/intensivo foi obtida apenas com o índice APNCU.

A partir do teste de Spearman, verificamos que as questões referentes ao componente processo de trabalho, es- pecificamente no que se refere à baixa cobertura de gestantes que cumpriram as metas propostas pelo PHPN, à realização dos exames básicos, à baixa realização de atividades de educação em saúde e ao baixo percentual de serviços que realizavam avaliação interna, foram as que contribuíram com significância estatística para que o pré-natal fosse classificado em maior proporção na categoria intermediário pelo Índice IPR/Pré-Natal. A análise comparativa interna das categorias dos índices para classificar o pré-natal quando associado a resultados indesejáveis do nascimento é apresentada na tabela 3 . Todas as razões de chances foram estatisticamente significativas, isto é, os intervalos de confiança não incluíram o valor 1,0 , exceto: para o índice APNCU na variável baixo peso ao nascer, ao comparar a categoria adequado com as categorias intensivo e intermediário; para o índice de Kessner, no resultado da prematuridade (adequado $\times$ inadequado); e na variável "ausência do aleitamento materno exclusivo" para esses dois índices nas categorias adequado $\times$ intensivo e adequado $\times$ intermediário, respectivamente.
Ainda de acordo com os dados expostos na tabela 3 para a variável "baixo peso ao nascer", os índices de Kessner e APNCU mostraram-se inconsistentes, já que o pré-natal adequado foi fator de risco para ocorrência desse desfecho em comparação às outras categorias.

Para a variável "prematuridade", observou-se que o Índice IPR/Pré-Natal, ao classificar o pré-natal como adequado, foi fator de proteção quando comparado à categoria intermediário. A mesma tendência foi observada para o índice de Kessner na comparação com a categoria intermediário e para o APNCU na comparação com as categorias intermediário e inadequado. Para este último, a prematuridade foi fator de risco quando a categoria adequado foi comparada a adequado superior/intensivo.

No que se refere ao peso insuficiente ao nascer, no Índice IPR/Pré-Natal a categoria adequado apresentou-se como fator de proteção quando comparada a intermediário. Essa mesma tendência foi observada para os índices de Kessner e APNCU.

Com relação à variável "sem aleitamento materno exclusivo", no IPR/ Pré-Natal menores chances de não haver aleitamento materno exclusivo foram observadas na categoria adequado em relação a intermediário. Entretanto, para os índices de Kessner e APNCU, a categoria adequado apresentou-se como fator de risco para este evento.

\section{DISCUSSÃO}

No que se refere às características da população do presente estudo, a média de idade das usuárias, assim como a faixa etária, a escolaridade e o número 
TABELA 3. Análise das categorias internas dos índices associadas a desfechos adversos do nascimento, João Pessoa (PB), Brasil, 2012

\begin{tabular}{|c|c|c|c|}
\hline Desfecho & Comparação & $\mathrm{OR}^{\mathrm{a}}$ & IC95\% ${ }^{\mathrm{b}}$ \\
\hline \multicolumn{4}{|c|}{ Baixo peso ao nascer $(<2500 \mathrm{~g})$} \\
\hline \multirow[t]{2}{*}{ Kessner } & Adequado vs. inadequado & $8,5094^{c}$ & 8,6425 a 8,6797 \\
\hline & Adequado vs. intermediário & $4,5125^{\mathrm{c}}$ & 4,4384 a 4,5872 \\
\hline \multirow[t]{3}{*}{ APNCU } & Adequado vs. intensivo & 1,1308 & 0,2465 a 8,8526 \\
\hline & Adequado vs. intermediário & $1,4284^{c}$ & 1,4023 a 1,4557 \\
\hline & Adequado vs. inadequado & 0,9300 & 0,0335 a 6,4490 \\
\hline IPR/Pré-Natal & Adequado vs. intermediário & 0,0000 & 0,0000 a 0,0000 \\
\hline \multicolumn{4}{|c|}{ Peso Insuficiente ao nascer (2 500 a $3000 \mathrm{~g}$ ) } \\
\hline \multirow[t]{2}{*}{ Kessner } & Adequado vs. inadequado & 0,0000 & 0,0000 a 0,0000 \\
\hline & Adequado vs. intermediário & $0,6730^{c}$ & 0,6487 a 0,6943 \\
\hline \multirow[t]{3}{*}{ APNCU } & Adequado vs. intensivo & $0,8459^{c}$ & 0,7195 a 0,9504 \\
\hline & Adequado vs. intermediário & $0,6734^{c}$ & 0,6501 a 0,6967 \\
\hline & Adequado vs. inadequado & 0,0000 & 0,0000 a 0,0000 \\
\hline IPR/Pré-Natal & Adequado vs. intermediário & $0,366^{c}$ & 0,1544 a 0,7944 \\
\hline \multicolumn{4}{|c|}{ Prematuridade ( $<37$ semanas) } \\
\hline \multirow[t]{2}{*}{ Kessner } & Adequado vs. inadequado & 1,6526 & 0,7707 a 3,7379 \\
\hline & Adequado vs. intermediário & 0,9139 & 0,9078 a 0,9167 \\
\hline \multirow[t]{3}{*}{ APNCU } & Adequado vs. intensivo & $2,0257^{c}$ & 2,7946 a 2,2875 \\
\hline & Adequado vs. intermediário & $0,7755^{c}$ & 0,6146 a 0,8142 \\
\hline & Adequado vs. inadequado & $0,8140^{c}$ & 0,8083 a 0,9976 \\
\hline IPR/Pré-Natal & Adequado vs. intermediário & $0,4230^{c}$ & 0,4101 a 0,4367 \\
\hline \multicolumn{4}{|c|}{ Sem aleitamento materno exclusivo } \\
\hline \multirow[t]{2}{*}{ Kessner } & Adequado vs. inadequado & 7,5332 & 7,5145 a $7,5532^{c}$ \\
\hline & Adequado vs. intermediário & 1,8765 & 0,9532 a 1,9841 \\
\hline \multirow[t]{3}{*}{ APNCU } & Adequado vs. intensivo & 5,9459 & 0,1195 a 7,2204 \\
\hline & Adequado vs. intermediário & 1,9365 & 1,9201 a $1,9534^{c}$ \\
\hline & Adequado vs. inadequado & 3,9897 & 1,9756 a $4,1741^{c}$ \\
\hline IPR/Pré-Natal & Adequado vs. intermediário & 0,5334 & 0,5147 a $0,5437^{c}$ \\
\hline
\end{tabular}

APNCU: Adequação da Utilização do Cuidado Pré-Natal. IPR: infra-estrutura, processo e resultados.

a $\mathrm{OR}=$ razão de chances.

b IC $95 \%$ = Intervalo de confiança de $95 \%$.

c Valores significativos.

de filhos estão em concordância com os achados de outros estudos $(3,26,27)$. É importante mencionar que, com relação à variável número de filhos, Ribeiro et al. (4) observaram que um número de filhos maior do que dois apresentou-se como fator de risco para o pré-natal inadequado. No entanto, quanto ao aspecto escolaridade, os dados encontrados no presente estudo foram superiores aos descritos por Mendonza-Sassi et al. (17), que observaram uma média de escolaridade de 6,6 $\pm 2,8$ anos. Para as gestantes classificadas com baixo peso, sobrepeso e obesidade, o ganho de peso apresentou-se inadequado quando comparado às faixas recomendadas pelo Institute of Medicine (IOM) (24). A respeito disso, mencionam-se os estudos de Barker (28) e Ludwig et al. (29), que apontaram a importância do ganho de peso gestacional adequado como bom preditor de peso ao nascer satisfatório e proteção contra riscos na vida adulta, indicativo de um pré-natal de qualidade.

Quanto à atenção ao pré-natal e aos resultados do nascimento (idade gestacional de início do pré-natal, total de consultas de pré-natal, idade gestacional no parto e peso ao nascer), os achados encontram-se em conformidade com a literatura especializada (16, 30-32). Para o tempo de aleitamento materno, os resultados mostraram-se superiores quando comparados à mediana nacional (33) e inferiores em relação aos achados de Agampodi et al. (34). É importante mencionar que os valores de média e mediana encontrados para o tempo de aleitamento materno exclusivo no atual estudo são considerados preocupantes, pois provavelmente demonstram a introdução precoce de alimentos desnecessários na dieta do bebê (35).

Quanto à análise de adequação do prénatal, assim como os achados do atual estudo, outros pesquisadores $(1,5,6)$ observaram diferenças na classificação da assistência pré-natal de acordo com o índice utilizado. Isso se justifica, pois os índices utilizam diferentes algoritmos para definir suas categorias de adequação do pré-natal. Tais diferenças podem resultar em conclusões diferentes quanto à situação real do pré-natal, levando a intervenções equivocadas (2). Bloch et al. (3) e VanderWeele et al. (5), em concordância com o observado neste estudo, relataram maior proporção de pré-natal adequado quando utilizado o índice de Kessner; enquanto isso, o pré-natal classificado como inadequado teve maior proporção pelo APNCU.

$\mathrm{O}$ alto percentual de pré-natal intermediário e a presença de pré-natal inadequado quando utilizados os índices de Kessner e APNCU podem ser justificados pelo critério desses índices de um número elevado de consultas, nove e 13 respectivamente, comparado ao critério de 6 consultas recomendado pelo Ministério da Saúde do Brasil (5, 15). Ressalta-se, ainda, que o pré-natal adequado-superior/intensivo observado pelo APNCU e a maior adequação pelo índice de Kessner podem representar o aumento do número de consultas e o início precoce do pré-natal ao longo dos anos pelas gestantes brasileiras (18).

Acerca da importância do processo de trabalho como maior contribuição para classificar o pré-natal como intermediário pelo Índice IPR/Pré-Natal, Nagahama et al. (16) apontam que esse componente é determinante para os resultados e reflete exatamente a essência da qualidade da atenção à saúde. Dessa forma, contribui para chamar a atenção sobre a necessidade de corrigir o curso das práticas de saúde.

A partir da análise interna das categorias dos índices, no caso da variável baixo peso ao nascer, os índices de Kessner e APNCU apresentaram inconsistência para detectar tal situação. Nesse contexto, especificamente quanto ao índice APNCU, Koroukian et al. (36), ao trabalharem com gestação de alto risco, também observaram que maior proporção de baixo peso ao nascer foi encontrada quando o pré-natal era classificado como adequado superior/intensivo em relação ao adequado. Os autores justificaram essa observação como um possível viés, ao considerar que o cuidado intensivo classificado pelo APNCU seria também destinado às gestantes em maior situação de risco, levando, dessa forma, a uma maior probabilidade de desfechos negativos nessa categoria. Porém, no atual estudo, esse viés deve ser desconsiderado, uma vez que as gestações foram de baixo risco. Portanto, as variáveis utilizadas pelo APNCU para classificar o pré-natal não são suficientemente sensíveis quando considerado o baixo peso ao nascer.

O baixo peso, assim como o peso insuficiente ao nascer, são os principais 
determinantes da morbimortalidade infantil (26). Quanto a esta última variável, destaca-se a consistência do Índice IPR/ Pré-Natal, no qual ter tido o pré-natal adequado proporciona menores chances de ocorrência desse agravo.

Para a variável prematuridade, o Índice IPR/Pré-Natal mostrou ser internamente consistente, pois realmente classificou os desfechos negativos na categoria de maior inadequação do pré-natal. Para os demais índices, os dados aqui encontrados estão em conformidade com o estudo de VanderWeele et al. (5), no qual os índices de Kessner e APNCU foram internamente consistentes quando associados a essa variável. Especificamente quanto ao Kessner, os resultados de nosso estudo divergiram dos relatados por esses pesquisadores, porém sem terem atingido significância estatística.

Na variável "sem aleitamento materno exclusivo", os índices comportaram-se de forma distinta. No Índice IPR/Pré-Natal, o pré-natal adequado foi um bom preditor de presença de aleitamento materno exclusivo. Ressalta-se que a amamentação é recomendada de forma exclusiva nessa fase da vida e reduz o risco de infecções gastrintestinais e respiratórias (34). Para os outros índices, houve inconsistência, já que o pré-natal adequado apareceu como fator de risco para a ocorrência desse desfecho.

Acrescentar novos critérios para avaliação da assistência pré-natal permite identificar o desempenho do serviço e evidencia a qualidade do cuidado, garantindo efetividade nos cuidados à gestante e reduzindo resultados obstétricos indesejáveis $(8,12,19)$. Portanto, o desenvolvimento e a aplicação do Índice IPR/Pré-Natal mostrou-se eficaz ao verificar que a classificação do pré-natal como adequado a partir de elementos de infra-estrutura, processo e resultados referentes aos serviços de saúde e usuárias teve associação com menor aparecimento de peso insuficiente ao nascer, prematuridade e ausência de aleitamento materno exclusivo.

Ressalta-se a inconsistência dos índices de Kessner e APNCU, pelos quais o pré-natal classificado como adequado foi fator de risco para a ocorrência de determinados desfechos obstétricos indesejáveis. Isso indica que, para a qualidade do pré-natal, aspectos mais abrangentes do que o número de consultas e a semana de início do pré-natal, conforme recomendam esses índices, devem ser considerados. Ainda, a partir do instrumento de coleta de dados, podem ser fornecidas características referentes aos serviços e usuárias de pré-natal da atenção primária. Esse tipo de informação pode contribuir substancialmente para os sistemas de saúde dos países, permitindo intervenções mais específicas e eficazes.

Conflitos de interesse. Nada declarado pelos autores.

\section{REFERÊNCIAS}

1. Heaman MI, Newburn-Cook CV, Green CG, Elliott LJ, Helewa ME. Inadequate prenatal care and its association with adverse pregnancy outcomes: a comparison of indices. BMC Pregnancy Childbirth. 2008;8:15. doi: 10.1186/1471-2393-8-15.

2. Beeckman K, Louckx F, Masuy-Stroobant G, Downe S, Putman K. The development and application of a new tool to assess the adequacy of the content and timing of antenatal care. BMC Health Serv Res. 2011;11:213. doi: 10.1186/1472-6963-11-213.

3. Bloch JR, Dawley K, Suplee PD. Application of the Kessner and Kotelchuck prenatal care adequacy indices in a preterm birth population. Public Health Nurs. 2009;26(5):449-59.

4. Ribeiro ER, Guimarães AM, Bettiol H, Lima DD, Almeida ML, de Souza L, et al. Risk factors for inadequate prenatal care use in the metropolitan area of Aracaju, Northeast Brazil. BMC Pregnancy Childbirth. 2009;9:31. doi: 10.1186/1471-2393-9-31.

5. VanderWeele TJ, Lantos JD, Siddique J, Lauderdale DS. A comparison of four prenatal care indices in birth outcome models: comparable results for predicting small-for-gestationalage. J Clin Epidemiol. 2009;62(4):438-45.

6. Alexander GR, Kotelchuck M. Quantifying the adequacy of prenatal care: a comparison of indices. Public Health Rep. 1996;111(5):408-18.

7. Kessner DM, Singer J, Kalk CW, Shlesinger ER. Infant death: an analysis by maternal risk and health care. Washington, DC: Institute of Medicine and National Academy of Sciences; 1973.

8. Morestin F, Bicaba A, Sermé J de D, Fournier P. Evaluating quality of obstetric care in low-resource settings: building on the literature to design tailor-made evaluation instru- ments-an illustration in Burkina Faso. BMC Health Serv Res. 2010;10:20. doi: 10.1186/ 1472-6963-10-20.

9. Donabedian A. An introduction to quality assurance in health care. Nova Iorque: Oxford University Press; 2003.

10. Kobayashi H, Takemura Y, Kanda K. Patient perception of nursing service quality: an applied model of Donabedian's structureprocess-outcome approach theory. Scand J Caring Sci. 2011;25(3):419-25.

11. Coulter I. The rocky road from efficacy to effectiveness: new research directions in CAM in the US. Em: Adams JE, ed. Examining the role of CAM in health care: linking researchers and practitioners. Brisbane: Network of Researchers in Public Health and Complementary and Alternative Medicine; 2009. Pp. 122-130.

12. Lee HC, Dudley RA, Gonzales R. Translating evidence into practice, policy, and public health in perinatal medicine. Neoreviews. 2011;12:e431-8.

13. Patton MQ, LaBossière $F$. L'évaluation axée sur l'utilisation. Em: Ridde V, Dagenais C. Approches et pratiques en évaluation de programme. Montreal: $1^{\prime}$ Université de Montréal; 2009.

14. Hartz ZMA, Silva LMV. Avaliação em Saúde: Dos modelos teóricos à prática na avaliação de programas e sistemas de saúde. Rio de Janeiro: Fiocruz; 2005.

15. Andreucci CB, Cecatti JG. Desempenho de indicadores de processo do Programa de Humanização do Pré-natal e nascimento no Brasil: uma revisão sistemática. Cad Saude Publica. 2011;27(6):1053-64.

16. Nagahama EEI, Santiago SM. O cuidado prénatal em hospital universitário: uma avaliação de processo. Cad Saude Publica. 2006;22(1): 173-9.

17. Mendoza-Sassi RA, Cesar JA, Teixeira TP, Ravache C, Araújo GD, Silva TC. Diferenças no processo de atenção ao pré-natal entre unidades da Estratégia Saúde da Família e unidades tradicionais em um município da Região Sul do Brasil. Cad Saude Publica. 2011; 27(4):787-96.

18. Brasil, Ministério da Saúde. Saúde Brasil 2009: uma análise da situação de saúde e da agenda nacional e internacional de prioridades em saúde. Brasília, DF: Ministério da Saúde; 2010. Disponível em: http://portal.saude.gov.br/ portal/arquivos/pdf/SAUDE_BRASIL_2009 COLETIVA.pdf Acessado em abril de 2013.

19. Beeckman K, Louckx F, Putman K. Determinants of the number of antenatal visits in a metropolitan region. BMC Public Health. 2010;10:527. doi: 10.1186/1471-2458-10-527.

20. Lodewyckx K, Peeters G, Spitz B, Blot S, Temmerman M, Zhang W, et al. Nationale richtlijn prenatale zorg: een basis voor een klinisch pad voor de opvolging van Zwangerschappen. Nederlandse Vereniging voor Obstetrie en Gynaecologie (NVOG); 2004. (KCE reports vol. 6A). Disponível em: https://kce. fgov.be/sites/default/files/page_documents/ d20041027313.pdf Acessado em maio de 2013.

21. Guide de surveillance de la grossesse. GyneWeb. 2007. Disponível em: http:/ /www. gyneweb.fr/sources/obstetrique/andem/ preambule.htm Acessado em maio de 2013.

22. Bolfarine H, Bussab W de O. Elementos de amostragem. São Paulo: Blucher; 2005.

23. Samico I, Hartz ZMA, Felisberto E, Carvalho EF. Atenção à saúde da criança: uma análise do grau de implantação e da satisfação de profissionais e usuários em dois municípios 
do estado de Pernambuco, Brasil. Rev Bras Saude Mater Infant. 2005;5(2):229-40.

24. Institute of Medicine (IOM). Nutrition during pregnancy. Washington, DC: National Academy Press; 1990.

25. Zupan J, Aahman E. Perinatal mortality for the year 2000: estimates developed by WHO. Genebra: WHO; 2005.

26. Hossain MI, Nahar B, Hamadani JD, Ahmed $\mathrm{T}$, Brown KH. Effects of community-based follow-up care in managing severely underweight children. J Pediatr Gastroenterol Nutr. 2011;53(3):310-9.

27. Xirasagar S, Fu JC, Liu J, Probst JC, Lin DP. Neonatal outcomes for immigrant vs. native-born mothers in Taiwan: an epidemiological paradox. Matern Child Health J. 2011;15(2): 269-79.

28. Barker DJ. Obesity and early life. Obes Rev. 2007;8 Suppl 1;45-9.

29. Ludwig DS, Currie J. The association between pregnancy weight gain and birthweight: a within-family comparison. Lancet. 2010; 376(9745):948-90.
30. Ayoola AB, Nettleman MD, Stommel M, Canady RB. Time of pregnancy recognition and prenatal care use: a population-based study in the United States. Birth. 2010;37(1): 37-43.

31. Victora CG, Matijasevich A, Silveira M, Santos I, Barros AJ, Barros FC. Socio-economic and ethnic group inequities in antenatal care quality in the public and private sector in Brazil. Health Policy Plan. 2010;25(4):253-61.

32. Nolan LA, Nolan JM, Shofer FS, Rodway NV, Emmett EA. The relationship between birth weight, gestational age and perfluorooctanoic acid (PFOA)-contaminated public drinking water. Reprod Toxicol. 2009;27(3-4):231-8.

33. Brasil, Ministério da Saúde, Secretaria de atenção a saúde, Departamento de Ações Programáticas e Estratégicas. II Pesquisa de Prevalência de Aleitamento materno nas Capitais Brasileiras e Distrito Federal 2009. Brasília: Ministério da Saúde; 2009. (Série C. Projetos, Programas e Relatórios). Disponível em: http://bvsms.saude.gov.br/bvs/publi cacoes/pesquisa_prevalencia_aleitamento_ materno.pdf Acessado em abril de 2013.

34. Agampodi SB, Agampodi TC, Piyaseeli UK. Breastfeeding practices in a public health field practice area in Sri Lanka: a survival analysis. Int Breastfeed J. 2007;2:13.

35. Vennemann MM, Bajanowski $T$, Brinkmann B, Jorch G, Yücesan K, Sauerland C, et al. Does breastfeeding reduce the risk of sudden infant death syndrome? Pediatrics. 2009; 123(3):e406-10.

36. Koroukian SM, Rimm AA. The "Adequacy of Prenatal Care Utilization" (APNCU) index to study low birth weight: is the index biased? J Clin Epidemiol. 2002;55(3):296-305.

Manuscrito recebido em 16 de setembro de 2012. Aceito em versão revisada em 12 de março de 2013.

ABSTRACT Objective. To develop and apply a new instrument to evaluate prenatal care based on the guidelines of the Brazilian Humanization Program for Prenatal Care and Birth, including quantitative and qualitative elements distributed according to the evalua-

Development and application of a new index for assessment of prenatal care

Key words tion triad of structure, process, and outcomes.

Methods. An analytic, descriptive, quantitative cross-sectional study was carried out with 238 women receiving care in 44 primary care services in the city of João Pessoa, Northeast Brazil, between November 2010 and December 2011. Physicians and nurses involved in prenatal care were also interviewed. The instrument developed by the investigators contains 23 questions relating to structure, process and outcomes of prenatal care. Based on the information collected, pre-natal care was classified according to an IPR/Pre-Natal index (Índice IPR/Pré-Natal, where I stands for infrastructure, P for work process and $\mathrm{R}$ for results). A value of 1 is attributed to each question if it complies with the criteria established for quality, or 2 if it does not comply. The percent of adequate answers in relation to the 23 total questions is used to classify prenatal care as: upper adequate ( $100 \%$ adequate answers); adequate $(\geq 75 \%)$; intermediate (51 to $74 \%)$; and inadequate ( $\leq 50 \%)$. The classification categories for the Pre-Natal/ IPR index were compared to those of the Kessner and Adequacy of Prenatal Care Utilization (APNCU) indices.

Results. Questions relating to the work process contributed significantly to the classification of prenatal care as intermediate according to the IPR/Pre-Natal. IPR/ Pre-Natal classification categories were consistent to detect prematurity, insufficient weight at birth and absence of exclusive breastfeeding.

Conclusions. The IPR/Pre-Natal index effectively incorporated quantitative and qualitative elements for the assessment of pre-natal care.

Prenatal care; primary care attention; birth weight; premature birth; breast feeding; Brazil. 